\title{
Adult-onset Still's disease in an elderly woman with atypical presentation
}

\author{
Takashi Kumano ${ }^{1}$, Hiromi I shibashi ${ }^{2}$, Yumiko Kubota ${ }^{3}$, Yasuhiro Ono ${ }^{4}$, Yoshifumi Tada ${ }^{5}$, Kohei \\ Nagasawa ${ }^{6}$, Masaya Yamaguchi ${ }^{4}$ \\ 1. Departments of Hematology and Oncology, Fukuoka Sanno Hospital, Fukuoka, Japan. 2. Department of Internal \\ Medicine, Fukuoka Sanno Hospital, Fukuoka, Japan. 3. Department of Dermatology, Fukuoka Sanno Hospital, Fukuoka, \\ Japan. 4. Department of Internal Medicine, Takagi Hospital, Okawa, Japan. 5. Department of Rheumatology, Faculty of \\ Medicine, Saga University, Saga, Japan. 6. Rheumatic Disease Center, Sawara Hospital, Fukuoka, Japan.
}

Correspondence: Takashi Kumano. Address: Departments of Hematology and Oncology, Fukuoka Sanno Hospital, 3-6-45 Momochihama, Sawara-ku, Fukuoka 814-0001, Japan. Email: tkumano@kouhoukai.or.jp

Received: February 6, 2014

DOI : $10.5430 /$ crim.v1n2p158
Accepted: May 28, 2014

Online Published: June 4, 2014

URL: http://dx.doi.org/10.5430/crim.v1n2p158

\section{Abstract}

We report an elderly case of adult-onset Still's disease (AOSD) with atypical presentation. The patient, an 80-year-old female, visited our hospital due to itchy linear urtical rash with low grade fever which occurred after taking a significant amount of blowfish milt four days before. Oral prednisolone of $30 \mathrm{mg}$ /day was successful. However, remittent fever appeared after reducing the prednisolone dose accompanied by the similar skin lesion. Hyperferritinemia was evident on the admission and the diagnosis of AOSD was made. We should keep in mind the presence of AOSD with atypical presentation in elderly patients.

\section{Keywords}

Adult-onset Still's disease, Elderly AOSD, Lichenoid plaque, Prednisolone, Hyperferritinemia

\section{I ntroduction}

Adult onset Still's disease (AOSD) is a rare inflammatory disorder of unknown etiology. AOSD usually affects young adults, and very few patients older than 70 -year-old have been reported ${ }^{[1-6]}$. Its clinical features include repeated high spike fever, arthralgia, lymphadenopathy, hepatosplenomegaly and an evanescent skin rash. A significant population of fever of unknown origin (FUO) is responsible for AOSD and this disease should be considered in the differential diagnosis of FUO. Early diagnosis is often difficult since no specific diagnostic marker is available, and its diagnosis is made by clinical symptoms and non-specific laboratory changes after the exclusion of infectious diseases, neoplasm and autoimmune diseases. Among such symptoms and laboratory changes, appearance of evanescent salmon-pink skin rash during febrile attack and hyperferritinemia are comparatively characteristic of AOSD. Approximately 80 to 90 percent of AOSD patients show this typical skin rash. We report here a rare case of elderly AOSD, whose cutaneous symptom and presentation were atypical. 


\section{Case report}

An 80-year-old Japanese female without significant past medical history except latent chronic HCV infection visited the dermatology department of our hospital due to apparent itchy linear urtical rash on the chest and back accompanied by low grade fever in February 2012. She had taken a significant amount of blowfish milt four days before. The dermatologist diagnosed this eruption to be toxicoderma, and $30 \mathrm{mg} /$ day of oral prednisolone was administered. Her eruption was soon ameliorated, and prednisolone was decreased to $10 \mathrm{mg} /$ day.

On day 8 , she complained of fever, sore throat and arthralgia at her cervical vertebra and bilateral shoulders. Her skin rash had become apparent again. During the febrile relapse, we closely re-evaluated underlying diseases. Her temperature was $37.5^{\circ} \mathrm{C}$, respiratory rate 18 breaths $/ \mathrm{min}$, pulse rate $90 \mathrm{beats} / \mathrm{min}$, and blood pressure $136 / 70 \mathrm{mmHg}$. Laboratory studies included WBC $9650 / \mu \mathrm{L}$ with $75.8 \%$ polys, RBC $433 \times 10^{6} / \mu \mathrm{L}$, Hgb $13.9 \mathrm{gm} / \mathrm{dL}$, Hct $41 \%$, MCV $94.7 \mathrm{fL}$, and platelet count $209 \times 10^{3} / \mu \mathrm{L}$. The sedimentation rate was elevated to $48 \mathrm{~mm} / \mathrm{hr}$, C-reactive protein (CRP) $2.23 \mathrm{mg} / \mathrm{dL}$, glucose 93 $\mathrm{mg} / \mathrm{dL}$, albumin $3.6 \mathrm{gm} / \mathrm{dL}$ BUN $16 \mathrm{mg} / \mathrm{dL}$, creatinine $0.64 \mathrm{mg} / \mathrm{dL}$, and calcium $9.4 \mathrm{mg} / \mathrm{dL}$. Total bilirubin, liver enzymes and procalcitonin (PCT) were not elevated, and anti-EBV and CMV IgG levels suggested latent exposures. Rheumatoid factor (RF) and anti-nuclear antibody (ANA) including other autoantibodies were negative and systemic autoimmune diseases seemed to be ruled out. Serum ferritin was elevated to $759 \mathrm{ng} / \mathrm{mL}$ (normal upper limit: $250 \mathrm{ng} / \mathrm{mL}$ ). Chest X-ray, abdominal ultrasound, echo-cardiography, chest and abdominal CT, and FDG-positron emission tomography (PET) revealed neither obvious infections nor tumors.

Antibiotics were added, but her fever did not improve. Considering these findings and her clinical course, we highly suspected that she was affected with AOSD. Then, we increased oral prednisolone from $10 \mathrm{mg} /$ day to $20 \mathrm{mg} /$ day with a combination of NSAIDs (etodolac $400 \mathrm{mg} /$ day). The administration was effective and her fever and arthralgia were getting improved. The CRP level, once increased to $6.50 \mathrm{mg} / \mathrm{dl}$, decreased to $2.96 \mathrm{mg} / \mathrm{dl}$. However, when we decreased the prednisolone dose to $7.5 \mathrm{mg} /$ day, the febrile attack to $39^{\circ} \mathrm{C}$ recurred and the itchy skin rash reappeared. Serum ferritin was elevated to $8008 \mathrm{ng} / \mathrm{ml}$, and liver enzymes, AST, ALT, and LDH, were elevated to 325U/L, 339U/L, and 644U/L, respectively, whereas the HCV-RNA level remained at low titers. From these events and findings we diagnosed her as having a relapse of AOSD, and continued the steroid therapy.

In the clinical course, pruritic lichenoid plaques had become apparent (see Figure 1). Skin biopsy of this plaques revealed dyskeratotic keratinocytes within the cornified layer and the superficial epidermis (see Figure 2). There were diffuse infiltrates of numerous neutrophils and lymphocytes in the dermis. Therefore, we considered these lichenoid plaques to be atypical skin lesions of AOSD, and continued the same dose of steroid therapy. Her febrile attack, arthralgia, liver damage, dermatological findings, and hyperferritinemia were gradually improved (see Figure 3). After eight weeks, her liver function was normalized, other laboratory data and skin lesions were improved and she is keeping well at outpatient for almost two years without prednisolone.

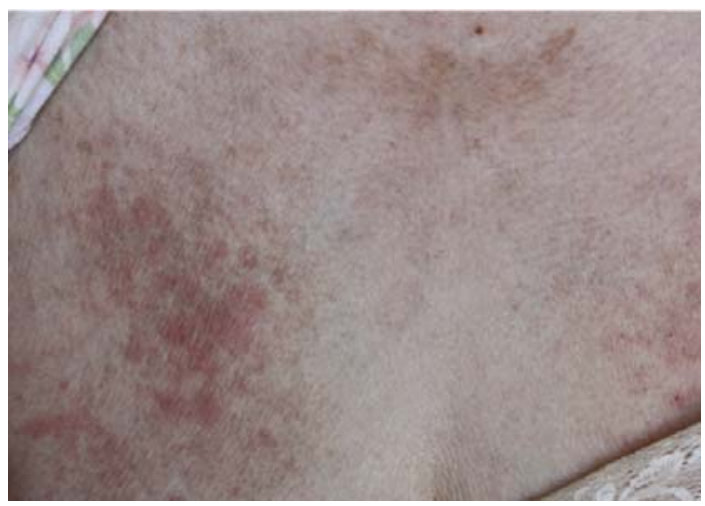

Figure 1. Persistent pruritic lichenoid plaques with somewhat linear appearance on anterior chest. 


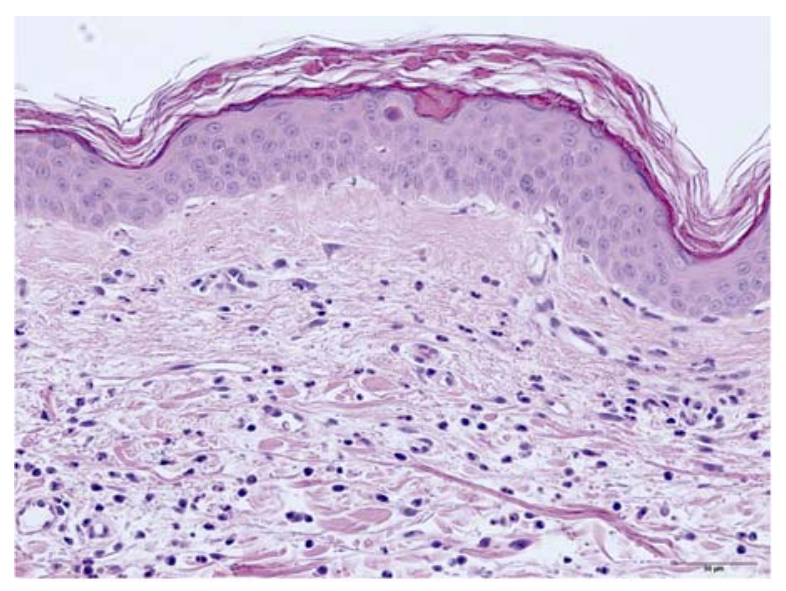

Figure 2. Histopathology of the skin lesion. Dyskeratotic keratinocytes are present within the cornified layer and the superficial epidermis. There are diffuse infiltrates of numerous neutrophils and lymphocytes in the dermis. Hematoxylin and eosin, original magnification $\times 100$.

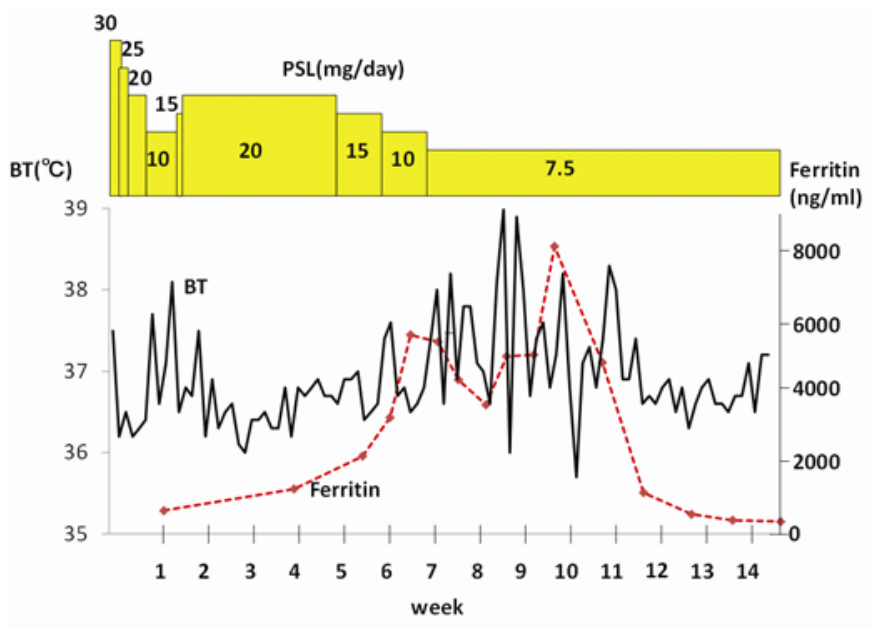

Figure 3. Clinical course. PSL: Prednisolone BT: Body Temperature

\section{Discussion}

AOSD is a systemic inflammatory condition of unknown etiology. No specific markers exist to aid diagnosis. After exclusion of infectious diseases, neoplasms and autoimmune conditions, AOSD is diagnosed based on clinical findings. The Yamaguchi's criteria ${ }^{[8]}$ is most widely cited in diagnosing AOSD. This criteria has about $96 \%$ of sensitivity and $92 \%$ of specificity. Though our case did not fully match the diagnostic criteria, it partially fulfilled three major criteria, and fully matched three minor criteria: persistent fever not over $39^{\circ} \mathrm{C}$, arthralgia not exceeding two weeks, atypical skin lesions, and leukocytosis approximately but not more than $10,000 / \mu \mathrm{L}$ in major symptoms, and positive sore throat and liver dysfunction, but negative for rheumatoid factor and anti-nuclear antibody in minor symptoms. However, she had remarkable hyperferritinemia, more than ten times of the normal value, and after exclusion of infectious, neoplastic, and autoimmune diseases, we diagnosed that she had affected with AOSD.

This case has several unique features as AOSD. The patient was very old as high as 80 years old, and her clinical presentation was atypical for AOSD. Epidemiologically, AOSD mainly affects young adult ${ }^{[9,10]}$, and only few cases older than 70 years old have been reported ${ }^{[1-6]}$. AOSD in elderly person sometimes present low-grade or atypical pattern of 
fever ${ }^{[7]}$. Furthermore, besides the characteristic fever pattern, rash and arthritis, atypical symptoms such as pleuritis, pneumonitis are sometimes present ${ }^{[6]}$.

Our patient's first symptom was a skin rash like toxicoderma which appeared after taking significant amount of blowfish milt four days before. AOSD patients often present at first such symptoms as fever (usually higher than $39^{\circ} \mathrm{C}$ ), and then evanescent skin rash during a febrile attack, lymphadenopathy, hepatosplenomegaly, sore throat, arthralgia, myalgia, arthritis, and serositis. In our case, patient initially presented low grade fever that was atypical for AOSD, and during the course, she had typical presentations such as high fever, sore throat, and arthralgia.

Evanescent, salmon-pink erythema predominantly involving trunk and proximal extremities, is a characteristic skin rash in AOSD patients, which is included as one of the diagnostic criteria ${ }^{[8]}$. However, her skin eruption was itchy urticarial rash like toxicoderma at first, and in the clinical course, it turned to persistent pruritic lichenoid plaque. Such skin lesions had been considered atypical for AOSD. However, recent findings show that not only typical evanescent rash but also various skin lesions are associated in AOSD ${ }^{[11-15]}$. The representative characteristic skin lesion among the non-classical skin rash is called persistent pruritic papules and plaques which presents erythematous, slightly scaly papules with linear configuration on the trunk and proximal extremities ${ }^{[12]}$. Other non-classical skin lesions include urticarial ${ }^{[13]}$. Interestingly, persistent pruritic papules and plaques show unique histological features such as peculiar, distinctive distribution of dyskeratotic keratinocytes in the cornified layers as well as in the epidermis ${ }^{[12,14]}$. Our dermatologist diagnosed her cutaneous symptom as toxicoderma at the initial presentation which was improved by the prednisolone administration. However, her persistent lichenoid plaques had appeared after steroid reduction. Skin biopsy of the patient revealed that these plaques were histologically consistent with AOSD, though to be somewhat different from typical biopsy findings of AOSD skin, in which perivascular infiltration of mononuclear and/or polymorphonuclear leukocytes in the dermis are seen.

In a broad sense, AOSD and juvenile rheumatoid arthritis(JRA) are now thought to be included in non-genetical autoinflammatory diseases, and as a trigger of the disease, infectious factors such as certain viruses including Epstein-Barr virus, cytomegalo-virus, hepatitis B and C, parvovirus B19 and bacteria have been proposed ${ }^{[16]}$. In this case, the patient had an HCV infection, however, the HCV RNA titer did not change during the clinical course and no other infections were documented. Some cases similar to drug hypersensitivity and the skin rash as urticaria have been reported ${ }^{[11]}$. In our case, hypersensitivity to blowfish milt might have triggered the onset of AOSD.

Treatment of patients with AOSD has been empirical, because there are no well-designed clinical trials to evaluate treatments. NSAIDs and corticosteroid are often used as first-line agents in the treatment of AOSD. Steroid therapy is effective in $76 \%-95 \%$ of patients ${ }^{[16]}$. Methotrexate and some immunosuppressive agents have shown their efficacy in steroid-refractory or steroid-dependent cases ${ }^{[17]}$. In cases refractory to above mentioned drug therapy, biological agents seem to achieve a better control of disease activity ${ }^{[18]}$. In our case, the sufficient doses of prednisolone $(0.6 \mathrm{mg} / \mathrm{kg})$ and NSAID were effective.

\section{Conclusions}

We have described a rare case of AOSD in an 80 years old female with atypical features and skin rash. The diagnosis was made by the exclusion of infection, neoplasm, and autoimmune diseases, and by the presence of atypical but characteristic clinical features including skin rash with markedly elevated serum ferritin levels. We should aware of the presence of ASOD with atypical presentation in elderly patients. Early recognition and an early treatment will save such patients. 


\section{References}

[1] Apostolova M, Sholb M, Glynn M. A rare presentation of adult onset Still's disease in an elderly patient. Rheumatology Reports. 2011; 3: e10. http://dx.doi.org/10.4081/rr.2011.e10

[2] Kurasawa M, Kotani K, Shida K, Yamada S, Tago T. Adult-onset Still's disease in a patient over 80 years old successfully treated with low-dose methotrexate therapy Age Ageing. 2007; 36: 104-106. http://dx.doi.org/10.1093/ageing/afl128

[3] Tamura K, Kubota K, Kurabayashi H Take H, Shirakura T. Elderly onset ofadult Still's disease: Report of a case. Clin Rheumatol. 1994; 13: 117-18. http://dx.doi.org/10.1007/BF02229879

[4] Ichiki H, Shishido M, Nisyiyama S. Two cases of adult onset of Still's disease in the elderly. Nihon Ronen Igakkai Zasshi. 1992; 29: 960-64. (in Japanese). http://dx.doi.org/10.3143/geriatrics.29.960

[5] Wouters JM, van Rijswijk MH, van de Putte LB. Adult onset Still's disease in the elderly: a report of two cases. J Rheumatol. 1985; 12: 791-93.

[6] Rubenstein EJ, Arkfeld DG. Adult Still's disease in a 75-year old patient. J Am Geriatr Soc. 2004; 52: 2144-45. http://dx.doi.org/10.1111/j.1532-5415.2004.52579_5.x

[7] Cagatay Y, Gul A, Cagatay A, Kamali S, Karadeniz A, et al (2009). Adult-onset Still's disease. Int J Clin Pract. 2009 ; 63: $1050-5$. http://dx.doi.org/10.1111/j.1742-1241.2007.01393.x

[8] Yamaguchi M, Ohta A, Tsunematsu T, Kasukawa R, Mizushima Y, et al. Preliminary criteria for classification of adult Still's disease. J Rheumatol. 1992; 19: 424-30.

[9] Ohta A, Yamaguchi M, Kaneoka H, Nagayoshi T, Hiida M. Adult Still's disease: Review of 228 cases from the literature. J Rheumatol. 1987; 14: 1139-46.

[10] van de Putte LB, Wouters JM. Adult-onset Still's disease. Bailliere's Clin Rheumatol. 1991; 5: 263-75. http://dx.doi.org/10.1016/S0950-3579(05)80283-3

[11] Woods MT, Gavino AC, Burford HN, Hughey SL, Mercado P, et al. The evolution of histopathologic findings in adult Still's disease. J Dermatopathol. 2011; 33: 736-739. http://dx.doi.org/10.1097/DAD.0b013e31820c6dd1

[12] Suzuki K, Kimura Y, Aoki M, Takezaki S, Tuchida T, et al. Persistent plaques and linear pigmentation in adult-onset Still's disease. Dermatology. 2001; 202: 333-335. http://dx.doi.org/10.1159/000051669

[13] Setterfield JF, Hughes GR, Kobza Black A. Urticaria as a presentation of adult Still's disease. Br J Dermatol. 1998 ; $138: 906-908$. http://dx.doi.org/10.1046/j.1365-2133.1998.02237.x

[14] Lee JY, Yang CC, Hsu MM. Histopathology of persistent papules and plaques in adult-onset Still's disease. J Am Acad Dermatol. 2005; 52: 1003-1008. http://dx.doi.org/10.1016/j.jaad.2005.02.032

[15] Lubbe J, Hofer M, Chavaz P, Saurat JH, Borradori L. Adult-onset Still's disease with persistent plaques. Br J Dermatol. 1999; 141: 710-713. http://dx.doi.org/10.1046/j.1365-2133.1999.03115.x

[16] Efthimiou P, Paik PK, Bielory L. Diagnosis and management of adult onset Still's disease. Ann Rheum Dis. 2006 ; 65: $564-572$. http://dx.doi.org/10.1136/ard.2005.042143

[17] Fujii T, Akizuki M, Kameda H, Matsumura M, Hirakata M, et al. Methotrexate treatment in patients with adult onset Still's disease-retrospective study of 13 Japanese cases. Ann Rheum Dis. 1997; 56(2): 144-148. http://dx.doi.org/10.1136/ard.56.2.144

[18] Suematsu R1, Ohta A, Matsuura E, Takahashi H, Fujii T, et al. Therapeutic response of patients with adult Still's disease to biologic agents: multicenter results in Japan. 2012. 